\title{
Improving contrast enhancement in magnetic resonance imaging using 5-aminolevulinic acid-induced protoporphyrin IX for high-grade gliomas
}

\author{
JUNKOH YAMAMOTO ${ }^{1}$, SHINGO KAKEDA ${ }^{2}$, TETSUYA YONEDA ${ }^{4}$, SHUN-ICHIRO OGURA ${ }^{5}$, \\ SHOHEI SHIMAJIRI ${ }^{3}$, TOHRU TANAKA ${ }^{6}$, YUKUNORI KOROGI ${ }^{2}$ and SHIGERU NISHIZAWA ${ }^{1}$
}

\begin{abstract}
Departments of ${ }^{1}$ Neurosurgery, ${ }^{2}$ Radiology and ${ }^{3}$ Surgical Pathology, University of Occupational and Environmental Health, Kitakyushu, Fukuoka 807-8555; ${ }^{4}$ Department of Medical Physics in Advanced Biomedical Sciences, Faculty of Life Sciences, Kumamoto University, Kumamoto 862-0976; ${ }^{5}$ Graduate School of Bioscience and Biotechnology, Tokyo Institute of Technology, Yokohama 226-8501; ${ }^{6}$ SBI Pharma Co., Ltd., Tokyo 106-6019, Japan
\end{abstract}

Received December 28, 2015; Accepted October 28, 2016

DOI: $10.3892 / 01.2016 .5539$

\begin{abstract}
Magnetic resonance imaging (MRI) with a gadolinium-based contrast agent is the gold standard for high-grade gliomas (HGGs). The compound 5-aminolevulinic acid (5-ALA) undergoes a high rate of cellular uptake, particularly in cancer cells. In addition, fluorescence-guided resection with 5-ALA is widely used for imaging HGGs. 5-ALA is water soluble, while protoporphyrin IX (PpIX) is water insoluble. It was speculated whether converting from 5-ALA to PpIX may relatively increase intracellular water content, and consequently, might enhance the T2 signal intensity in HGG. The aim of the present study was to assess whether 5-ALA-induced PpIX enhances the T2 signal intensity in patients with HGGs. A total of 4 patients who were candidates for HGG surgical treatment were prospectively analyzed with preoperative MRI. Patients received oral doses of 5-ALA $(20 \mathrm{mg} / \mathrm{kg}) 3 \mathrm{~h}$ prior to anesthesia. At $2.5 \mathrm{~h}$ post-5-ALA administration, T2-weighted images (T2WIs) were obtained from all patients. Subsequently, tumors were evaluated via fluorescence using a modified operating microscope. Fluorescent tumor tissues were obtained to analyze the accumulation of 5-ALA-induced PpIX within the tumors, which was confirmed quantitatively by high-performance liquid chromatography (HPLC) analysis. The MRI T2 signal intensity within the tumors was evaluated prior to and following 5-ALA administration. Three glioblastoma multiformes (GBMs) and 1 anaplastic oligodendroglioma
\end{abstract}

Correspondence to: Dr Junkoh Yamamoto, Department of Neurosurgery, University of Occupational and Environmental Health, 1-1 Iseigaoka, Yahatanishi-ku, Kitakyushu, Fukuoka 807-8555, Japan

E-mail: yama9218@med.uoeh-u.ac.jp

Key words: 5-aminolevulinic acid, glioma, magnetic resonance imaging, nephrogenic systemic fibrosis, cancer, contrast reagent, drug repositioning
(AO) were included in the analysis. Intraoperatively, all GBMs exhibited strong fluorescence of 5-ALA-induced PpIX, whilst no fluorescence was observed in the AO sample. HPLC analysis indicated a higher accumulation of 5-ALA-induced PpIX in the GBM samples compared with the AO sample. In total, 48 regions of interest were identified within the tumors from T2-WIs. In the GBM group, the relative T2 signal intensity value within the tumors following 5-ALA administration was significantly increased compared with the T2 signal intensity value prior to 5-ALA administration $(1.537 \pm 0.021$ and $1.577 \pm 0.023$, respectively; $\mathrm{P}=0.0055)$. No significant differences were observed in the AO group. These results suggest that the 5-ALA-induced PpIX enhanced the T2 signal intensity in HGG. Therefore, 5-ALA may be a potentially useful MRI contrast reagent for $\mathrm{HGG}$.

\section{Introduction}

High-grade gliomas (HGGs), such as glioblastoma multiforme (GBM), are highly aggressive primary brain tumors and locally invasive into surrounding normal brain tissues (1). For the multimodal treatment of GBM, cytoreductive surgery is essential (4). The extent of tumor resection [starting from $78 \%$ and increasing to nearly $100 \%$ of gadolinium-based contrast-enhanced tumor volume on magnetic resonance imaging (MRI)] improves the overall survival of patients with GBM (5). Subsequently, post-operative adjuvant radiotherapy is required for managing invaded residual tumors in HGGs (6). However, despite the availability of multimodal treatment like surgery and radiochemotherapy, the mean survival of patients with GBM remains at only 12-14 months (2,3), and the recurrence rate of GBM is nearly $100 \%$ (1). Therefore, novel treatment concepts for HGGs are necessary. Conventional MRI, including contrast-enhanced T1-weighted imaging (CE-T1WI), is the gold standard to evaluate tumor extension, post-operative residual tumors and treatment responses after chemo-radiation for HGGs (7). Contrast enhancement is able to identify vital tumor tissue due to gadolinium-based contrast agent (GBCA) leakage. This is a result of the abnormal permeability of 
the blood-brain barrier (BBB) caused by an abundance of dysfunctional tumor vessels in HGGs $(8,9)$. However, a highly infiltrative HGG tumor burden is not consistently associated with abnormal BBB permeability (10). Previous pathological studies have revealed that glioma cells can infiltrate the brain parenchyma far beyond the contrast-enhanced lesions $(9,11)$. Thus, conventional radiological investigations using GBCA may significantly underestimate the extent of HGG tumor growth, particularly in cases of highly infiltrative HGGs (9). In addition, GBCA administration in patients with renal dysfunction is limited in order to avoid the development of nephrogenic systemic fibrosis (12). Novel candidate MRI contrast reagents have not yet been identified as alternatives to GBCA.

5-aminolevulinic acid (5-ALA) is a natural biochemical precursor of heme (13). In cancer cells, 5-ALA is metabolized into protoporphyrin IX (PpIX), which functions as a photosensitizer, following systemic administration (14-16). Specifically, 5-ALA induces a high accumulation of PpIX in glioma cells (17). Therefore, fluorescence-guided resection using 5-ALA in HGG treatment has been useful in determining tumor borders, making tumor resection easier compared with conventional microsurgery (18). 5-ALA is water soluble, while PpIX is water insoluble (19). It has been speculated that converting 5-ALA to PpIX within glioma cells may increase the intracellular free water content, and consequently induce an enhancement of the T2 signal intensity. Therefore, in the present study, a prospective assessment of the effect of 5-ALA-induced PpIX on MRI T2 signal intensity in patients with HGGs was performed. In addition, the potency of 5-ALA-induced PpIX as an enhancer of MRI T2 signal intensity for HGGs is discussed.

\section{Materials and methods}

Study design. A prospective case study design was used, and included eligible patients from September 2014 to February 2015 who gave written informed consent. All patients underwent surgical treatment at the Department of Neurosurgery, The Affiliated Hospital of the University of Occupational and Environmental Health (Kitakyushu, Japan). The study was approved by the institutional review board of the University of Occupational and Environmental Health (approval no. H26-075). The primary inclusion criteria were cases of suspected HGG as determined by preoperative MRI, with planned surgical resection, no contraindications for 5-ALA, and informed patient consent. The exclusion criteria included recurrent $\mathrm{HGG}$, patient age of $<20$ years old and treatment history for other brain tumors.

Study protocol. All MRIs were performed using a 3.0-T unit (Signa Excite; GE Medical Systems, Milwaukee, Wisconsin) with a dedicated 8-channel phased-array coil (GE Medical Systems). All patients underwent brain MRI scans both prior to and following 5-ALA administration.

Patients underwent the institution's standard brain MRI protocol for HGG, including an axial T2WI, a T1WI and a CE-T1WI. The following imaging parameters were used for axial T2WI: a TR/TE of 4,000/85; a flip angle of $90^{\circ}$; a bandwidth of $62.5 \mathrm{kHz}$; a section thickness of $3 \mathrm{~mm}$; a matrix of $512 \times 224$; a field of view (FOV) of $18 \times 18 \mathrm{~cm}$; and an imaging time of 2 min $16 \mathrm{sec}$. For CE-T1WI, the following imaging parameters were used following administration of the contrast material. CE-T1WI (spin-echo) was acquired with a section thickness of $3 \mathrm{~mm}$; a FOV of $18 \mathrm{~cm}$; a matrix of $224 \times 224$; an imaging time of $2 \mathrm{~min} 40 \mathrm{sec}$; and 2 acquired excitations. A three-dimensional fast spoiled gradient-echo (3D fast SPGR) was acquired with the parameters of 10/4.1/700/7 min $20 \mathrm{sec}$ [repetition time $(\mathrm{ms}) / e c h o$ time $(\mathrm{ms}) /$ inversion time/imaging time], a flip angle of $10^{\circ}$, a $24 \mathrm{~cm}$ FOV, a $512 \times 256$ matrix, and $1.4 \mathrm{~mm}$ thick sections with a $2.5 \times 2.5 \times 8 \mathrm{~mm}$ resolution. The 3D fast SPGR data were reconstructed in the sagittal and coronal planes. For all patients, gadodiamide hydrate (Omniscan; Daiichi Pharmaceutical, Tokyo, Japan) or gadopentetate dimeglumine (Magnevist; Bayer Schering-Pharma, Berlin, Germany) was administered at a dose of $0.1 \mathrm{mmol} / \mathrm{kg}$ via intravenous bolus injection. Conventional MRI scans were obtained from all patients at a maximum of 8 days prior to surgery.

5-ALA was prepared by dissolving the compound in $50 \mathrm{ml}$ of water $(20 \mathrm{mg} / \mathrm{kg})$, which was immediately administered orally to each patient $3 \mathrm{~h}$ prior to anesthesia as previously described (18). T2W Is were obtained $\sim 2.5 \mathrm{~h}$ post-5-ALA administration. Subsequently, patients immediately underwent surgery, performed by two skilled neurosurgeons (Department of Neurosurgery, University of Occupational and Environmental Health). Patient registration in the neuronavigation system was performed using automatic registration tools (BrainLab AG, Feldkirchen, Germany) based on pre-operative CE-T1WIs. To avoid photobleaching of 5-ALA-induced PpIX due to exposure to the microscope's light, surgeons confirmed that the brain tumors corresponded to $\mathrm{CE}$ lesions on the neuronavigation system, and immediately evaluated the fluorescence of the 5-ALA-induced PpIX within the tumors. The 5-ALA fluorescence was graded as 'none', 'vague', or 'strong' as previously described (20). 'Strong' fluorescence was characterized as vivid red, while 'vague' fluorescence was defined as less vivid pink. Intraoperatively, each patient was evaluated for their fluorescence status (none, vague or strong) by two independent observers. Fluorescent tumor specimens were obtained safely and immediately snap-frozen in liquid nitrogen and stored at $-80^{\circ} \mathrm{C}$ for HPLC analysis. Simultaneously, tumor specimens were also obtained for histopathology. No corticosteroids were administered to any patients prior to obtaining tumor specimens.

HPLC analyses for 5-ALA-induced PpIX in tumor specimens. Accumulation of 5-ALA-induced PpIX in HGGs was confirmed using HPLC analyses. Using a previously described HPLC analysis method with porphyrin metabolites (21), tumor specimens ( $1 \mathrm{~mm}$ in diameter) were treated with $200 \mu \mathrm{l}$ of $0.1 \mathrm{M} \mathrm{NaOH}$ and homogenized on ice with a PowerMaster II (Array Solutions, Sunnyvale, TX, USA). Tumor specimens consisted of at least 2 samples from each patient. Aliquots $(10 \mu \mathrm{l})$ of $\mathrm{NaOH}$-treated samples were transferred to a protein concentration assay (Quick Start ${ }^{\mathrm{TM}}$ Bradford Dye Reagent, Bio-Rad Laboratories, Inc., Hercules, CA, USA), while the remaining $50 \mu \mathrm{l}$ was denatured by the addition of N,N-dimethylformamide: Isopropanol (100:1, v/v) solution added at $3 \mathrm{X}$ the sample volume $(150 \mu \mathrm{l})$. Following overnight storage in the dark, the prepared samples were subjected to HPLC analysis as previously described $(13,22)$ with the following modifications. 
Briefly, the porphyrins were separated using the Prominence HPLC system (Shimadzu, Kyoto, Japan), which was equipped with a reversed-phase C18 column, SG300, $5 \mu \mathrm{m}, 4.6 \times 250 \mathrm{~mm}$; (CAPCELL PAK, Shiseido, Tokyo, Japan) and maintained at $40^{\circ} \mathrm{C}$. The elution solvents were solvent A (1 M ammonium acetate, including $12.5 \%$ acetonitrile, $\mathrm{pH}$ 5.2) and solvent B (50 mM ammonium acetate, including $80 \%$ acetonitrile, $\mathrm{pH}$ 5.2). The elution was first performed with solvent $A$ for 5 min and subsequently with a linear gradient of solvent B (0-100\%) for $25 \mathrm{~min}$, followed by an elution with solvent B for $10 \mathrm{~min}$. The elution flow was maintained at a constant rate by using a fluorospectrometer (excitation at $404 \mathrm{~nm}$, detection at $624 \mathrm{~nm}$ ). The porphyrin concentrations in the samples were estimated using calibration curves obtained with standard porphyrins.

Image analysis. It was hypothesized that 5-ALA-induced PpIX would induce changes in the T2 signal intensity in HGGs as 5-ALA is water soluble, while PpIX is water insoluble (19). Therefore, an experienced neuroradiologist was blinded to the clinical information (the intraoperative findings of the 5-ALA-induced PpIX fluorescence and pathological diagnosis). The neuroradiologist reviewed the T2WI pre- and post-5-ALA administration in each patient. According to the CE lesion on the CE-T1WI (which was defined as a tumor) obtained prior to 5-ALA administration, an axial slice with a maximum amount of tumor was defined as the standard slice. Three slices, which included the standard slice and adjacent slices above and below the standard slice, were used for the axial T2WI evaluations. Similar to previous studies (23-25), the regions of interest (ROI) for the signal intensity analyses were drawn directly on the T2WI. Briefly, all images, including the T2WI and the CE-T1WI prior to and following 5-ALA administration, were displayed on a diagnostic monitor (Flexscan L365; Eizo Nanao Corporation, Ishikawa, Japan) simultaneously. All ROIs were an arbitrarily chosen, uniform shape and size (elliptical, $50 \mathrm{~mm}^{2}$ ). The ROIs were identified within tumors on the T2WI, and corresponded to the CE lesion on the CE-T1WI in 4 spots in each slice. Therefore, a total of 12 ROIs were assessed in each patient pre- and post-5-ALA administration. The ROI placement avoided areas of necrosis, hemorrhage, calcification, and engorged vessels, in accordance with other sequences of MRI and computed tomography (CT) $(23,25)$. ROIs were also placed within the normal white matter of the contralateral brain region (the contralateral cerebellar peduncle in the cerebellar glioma). To avoid scaling problems on each MRI scan image, signal intensity ratios were calculated for all images by using the following formula: Signal intensity ratio $=$ signal intensity of tumor $/$ signal intensity of normal white matter (23).

Histopathology. A pathologist from the University of Occupational and Environmental Health performed the subsequent histopathology. Tumor specimens for histological assessment were obtained during surgery according to the imaging results on the neuronavigation system and the 5-ALA fluorescence, and were immediately fixed in $10 \%$ neutral buffered formalin for $24 \mathrm{~h}$ at room temperature. Then, tumor specimens embedded in paraffin were cut systematically at $4 \mu \mathrm{m}$ thickness using a sliding microtome (Leica SM2010R;
Leica Microsystems, Wetzler, Germany) for staining with hematoxylin and eosin, followed by immunohistochemistry. All immunohistochemical stainings were carried out using Dako Envision kit (Dako, Glostrup, Denmark) according to the manufacturer's protocol, and using the following commercially available antibodies: Anti-glial fibrillary acidic protein (dilution, 1:500; \#Z0344; Dako), Anti-Oligo2 (dilution, 1:20; \#18953; Immuno-Biological Laboratories Co., Ltd., Fujioka, Japan), anti-S100 (dilution, 1:300; \#Z0311; Dako), anti-IDH1 R132H (dilution, 1:20; \#DIA-H09; Dianova GmbH, Hamburg, Germany), anti-p53 (dilution, 1:50; \#M7001; Dako) and anti-Ki-67 (dilution, 1:150; \#M7240; Dako). The slides were incubated with the primary antibodies for $30 \mathrm{~min}$ at room temperature. HGGs were classified neuropathologically according to the World Health Organization classification of tumors of the central nervous system (26).

Statistical analysis. Statistical analyses were performed using StatView 5.0 (SAS Institute Inc., Cary, North Carolina). T2 signal intensity was compared prior to and following 5-ALA administration using a paired $t$-test Accumulation of 5-ALA-induced PpIX was analyzed with an unpaired $t$-test. The data are presented as the mean \pm standard error. $\mathrm{P}<0.01$ was considered to indicate a statistically significant difference.

\section{Results}

Patient characteristics. Surgery was performed on 9 patients with suspected HGGs, which were identified from preoperative MRIs from September 2014 to February 2015. Of these, 5 patients (recurrent cases, $n=4$; child case, $n=1$ ) were excluded according to the exclusion criteria. Therefore, 4 patients ( 3 male, 1 female; mean age, $60 \pm 18.1$ years) were included in this study. Patient demographics are summarized in Table I. A total of 3 patients were pathologically diagnosed with glioblastoma multiforme (GBM) and 1 with anaplastic oligodendroglioma (AO). The tumors were located in the right frontal lobe in $2 \mathrm{GBM}$ cases, the right cerebellar hemisphere in $1 \mathrm{GBM}$ case and the right cingulate gyrus in the AO case. The CE-T1WI exhibited a heterogeneous and irregularly thick margin in patients with GBM, and focally enhanced heterogeneity in the patient with AO. The mean duration of the MRI examination (from initial MRI to surgery and post-5-ALA administration MRI) was 4 days.

Intraoperative observations and 5-ALA-induced PpIX accumulation. The intraoperative findings for the 5-ALA fluorescence were 'strong' in all 3 GBM cases and 'none' in the AO case. The quantitative HPLC analysis demonstrated that the amount of 5-ALA-induced PpIX in tumors of 'strong'/GBM group was $113.1 \pm 32.6 \mathrm{pmol} / \mathrm{mg}$ of protein. The 5-ALA-induced PpIX was not detected in the tumor of the 'none'/AO group $(<0.1 \mathrm{pmol} / \mathrm{mg})$. The former group tended to produce higher 5-ALA-induced PpIX values than the latter, although no statistically significant differences were identified $(\mathrm{P}=0.2927)$.

Evaluation of T2 signal intensity prior to and following 5-ALA administration. In total, 48 within-tumor ROIs were obtained from the T2WI prior to and following 5-ALA 
Table I. Clinical characteristics of high-grade gliomas.

\begin{tabular}{|c|c|c|c|c|c|c|c|}
\hline Case & $\begin{array}{l}\text { Age, } \\
\text { years }\end{array}$ & Gender & $\begin{array}{l}\text { Pathological } \\
\text { diagnosis }\end{array}$ & Tumor location & $\begin{array}{l}\text { MRI findings } \\
\text { (CE-T1WI) }\end{array}$ & $\begin{array}{c}\text { Duration from } \\
\text { initial MRI } \\
\text { to surgery }\end{array}$ & $\begin{array}{c}\text { PpIX } \\
\text { accumulation } \\
\text { intraoperative } \\
\text { findings }\end{array}$ \\
\hline 1 & 61 & M & GBM & Right frontal lobe & Irregular, enhanced thick margin & 5 days & Strong \\
\hline 2 & 63 & $\mathrm{~F}$ & GBM & Right frontal lobe & Wholly enhanced, heterogeneous & 1 days & Strong \\
\hline 3 & 36 & M & $\mathrm{AO}$ & Right cingulate gyrus & Focally enhanced, heterogeneous & 8 days & None \\
\hline 4 & 80 & M & GBM & $\begin{array}{l}\text { Right cerebellar } \\
\text { hemisphere }\end{array}$ & Irregular, enhanced thick margin & 2 days & Strong \\
\hline
\end{tabular}

GBM, glioblastoma multiforme; AO, anaplastic oligodendroglioma; MRI, magnetic resonance imaging; CE-T1WI, contrast enhanced T1 weighted imaging; PpIX, protoporphyrin IX.

administration. In the 'strong' group, the relative value of the T2 signal intensity within the tumors post-5-ALA administration was significantly higher than the signal intensity value prior to 5-ALA administration $(1.537 \pm 0.021$ and $1.577 \pm 0.023$, respectively; $\mathrm{P}=0.0055)$. By contrast, the value of the $\mathrm{T} 2$ signal intensity in the 'none' group did not differ significantly between tumors obtained prior to or following 5-ALA administration $(1.551 \pm 0.039$ and $1.572 \pm 0.040$ respectively; $\mathrm{P}=0.1281$ ) (Table II).

Representative cases. A 61-year-old healthy male presented with left hemiparesis and cerebral cortical dysfunction (case 1). MRI revealed an irregular, enhanced mass with peritumoral brain edema in the right frontal lobe (Fig. 1A and B). The patient underwent surgery 5 days later. The T2WI post-5-ALA administration and prior to surgery exhibited a slightly higher signal within the tumor compared with the T2WI prior to 5-ALA administration (initial MRI) (Fig. 1C). Intraoperative findings demonstrated a 'strong' 5-ALA fluorescence within the tumor (Fig. 1D and E). Histopathological examination identified hypercellularity, marked nuclear atypia and prominent vascular proliferation consistent with GBM (Fig. 1F).

A 63-year-old woman presented with left hemiparesis (case 2). An MRI scan revealed a heterogeneously enhanced mass in the right frontal lobe with peritumoral brain edema (Fig. 2A and B). The patient underwent surgery the following day. Prior to surgery, the T2WI following 5-ALA administration exhibited a slightly increased T2 signal within the tumor compared with the T2WI prior to 5-ALA administration (Fig. 2C). Intraoperative findings demonstrated a 'strong' 5-ALA fluorescence within the tumor (Fig. 2D and E). Histopathological examination confirmed the diagnosis of GBM (Fig. 2F).

\section{Discussion}

The present study demonstrated that 5-ALA-induced PpIX enhanced the T2 signal intensity in HGGs. In particular, GBM with a high accumulation of PpIX had an increased T2 signal intensity within the tumors compared with $\mathrm{AO}$, which had a low accumulation of PpIX. To the best of our knowledge, the present study is the first to assess the effects of 5-ALA-induced PpIX on MRI signaling, in particular in T2WI in clinical HGG cases.
Table II. Changes in T2 signal intensity before and after 5-ALA administration.

\begin{tabular}{lccc}
\hline & & \multicolumn{2}{c}{$\begin{array}{c}\text { Relative signal } \\
\text { intensity value (T2) }\end{array}$} \\
\cline { 3 - 4 } PpIX fluorescence & 5-ALA & Mean \pm SE & P-value \\
\hline Strong $(\mathrm{n}=36)$ & Pre & $1.537 \pm 0.021$ & 0.0055 \\
& Post & $1.577 \pm 0.023$ & \\
None $(\mathrm{n}=12)$ & Pre & $1.551 \pm 0.039$ & 0.1281 \\
& Post & $1.572 \pm 0.040$ & \\
\hline
\end{tabular}

PpIX, porphyrin IX; 5-ALA, 5 aminolevulinic acid.

Several studies have demonstrated that pre-treatment CE-T1WI tumor volume, early CE-T1WI tumor volume changes, the treatment response criteria and residual CE-T1WI volumes in the early follow up MRI scans may serve as predictors for both progression free survival and overall survival in HGGs (27-30). Bevacizumab is a well-known vascular endothelial growth factor inhibitor, which functions as a powerful corticosteroid to decrease the permeability of the BBB in HGGs (31). Bevacizumab is able to induce an initially high radiographic response rate, with a profound reduction in GBCA enhancement in MRI scans obtained as soon as $24 \mathrm{~h}$ after the first bevacizumab dose in HGG cases (27). However, this rapid decrease in the degree of contrast enhancement may not represent the true changes in the cellular burden or tumor biology (32). Therefore, certain studies have attempted to predict the bevacizumab response in HGGs using subtraction maps of CE-T1WI, or modified relative cerebral blood volume imaging based on GBCA administration $(32,33)$. Metabolic imaging using positron emission tomography (PET) amino acid radiolabeled tracers may overcome certain disadvantages of MRI, as the uptake of an amino acid tracer occurs primarily independently of the regional tumor perfusion and $\mathrm{BBB}$ permeability. Therefore, this technique may more accurately estimate the tumor size and extension of the metabolically active tumor (34-37). However, the spatial resolution of PET images is extremely low compared with MRI. In addition, the 

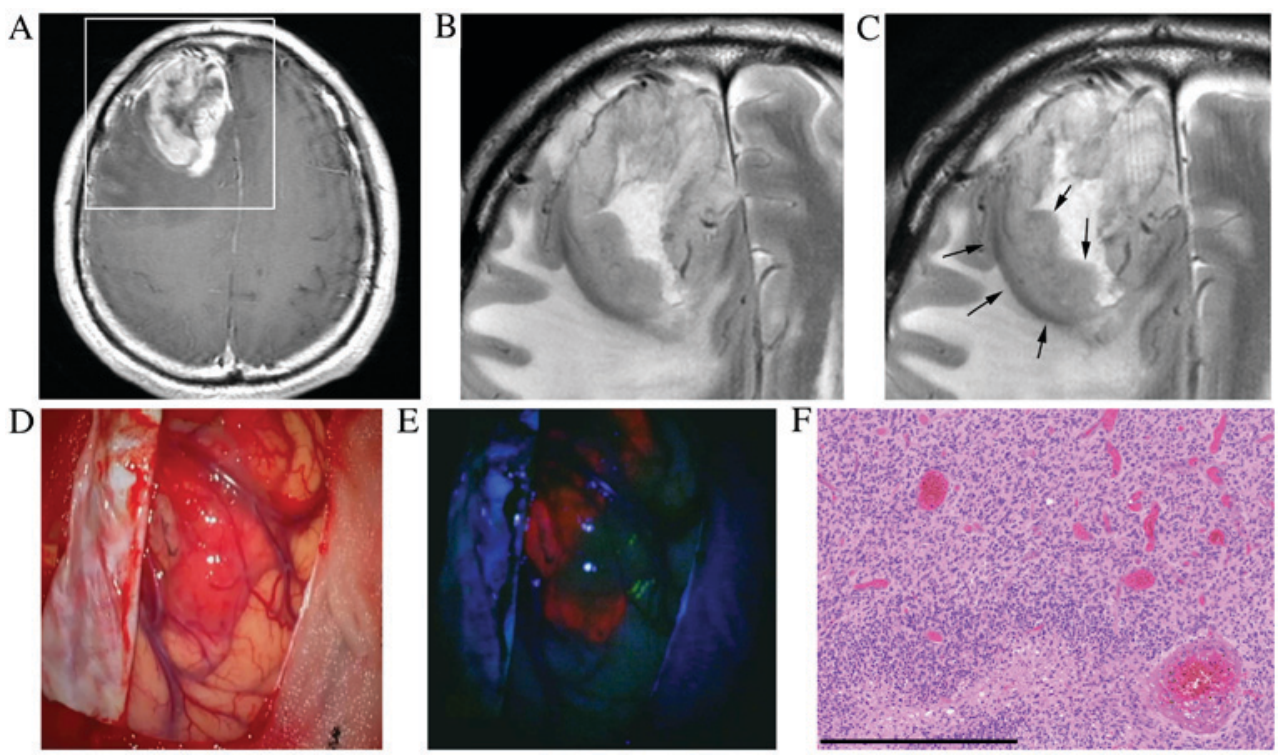

Figure 1. Glioblastoma in a 61-year-old male patient (case 1). (A) The axial contrast enhanced-T1 reveals an irregular, enhanced mass with peritumoral brain edema in the right frontal lobe. A magnified axial view of the T2-weighted image in (B) prior to (C) and following 5-ALA administration. The solid component of the tumors demonstrated a slight enhancement in the T2 signal following 5-ALA administration (arrows in C). Intraoperative findings of the surface of the right frontal lobe under (D) white and (E) violet-blue light. 5-ALA tumor fluorescence was strongly observed though the thinning brain cortex, which covered the tumor. (F) Histopathological findings included hypercellularity, marked nuclear atypia and prominent vascular proliferation consistent with glioblastoma multiforme. Staining, hematoxylin and eosin; scale bar, $400 \mu \mathrm{m}$. 5-ALA, 5 aminolevulinic acid.
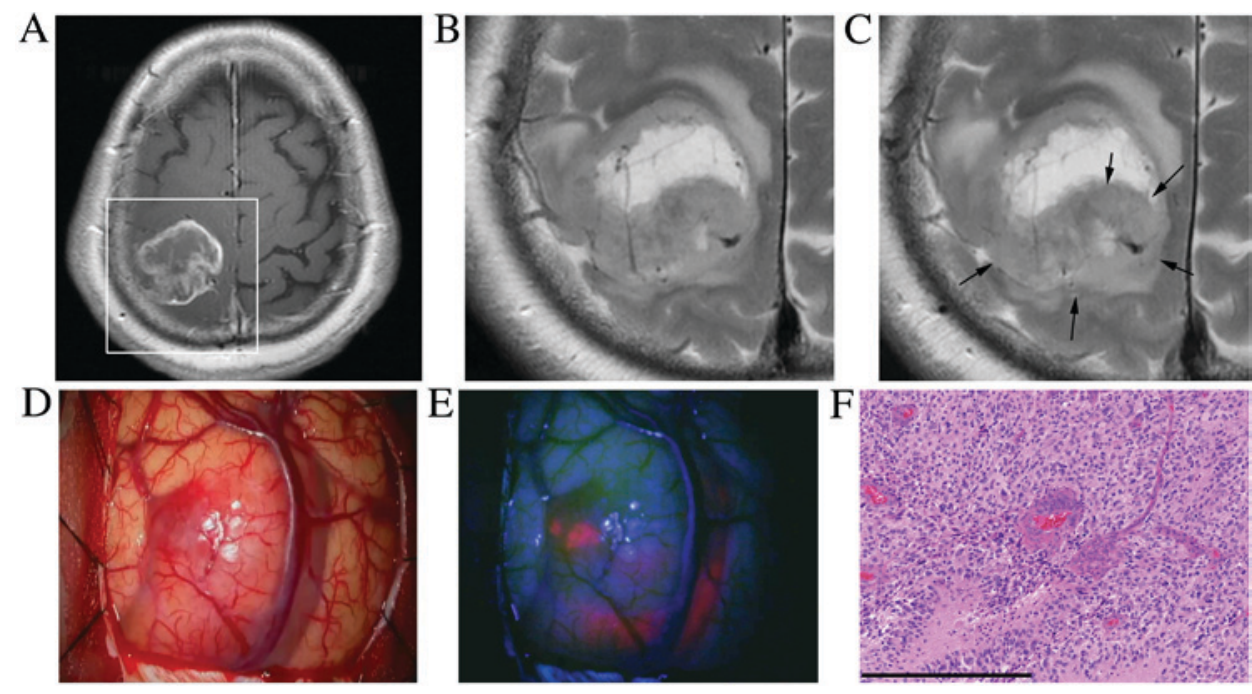

Figure 2. Glioblastoma in a 63-year-old female patient (case 2). (A) Axial contrast enhanced-T1 reveals a heterogeneously enhanced mass in the right frontal lobe with peritumoral brain edema. A magnified axial view of the T2WI (B) prior to and (C) following 5-ALA administration. The solid component of the tumors demonstrated slight enhancement of the T2 signal following 5-ALA administration (arrows in C). Intraoperative findings of the surface of the right frontal lobe under (D) white and (E) violet-blue light. 5-ALA tumor fluorescence was strongly observed though the thinning brain cortex, which covered the tumor. (F) Histopathological findings were consistent with glioblastoma multiforme. Staining, hematoxylin and eosin; scale bar, $400 \mu \mathrm{m}$. 5-ALA, 5 aminolevulinic acid.

general use of the PET system is also low compared with MRI due to equipment location and the handling of radiolabeled reagents. Therefore, metabolic imaging with high spatial resolution, such as MRI, is required for HGG management. In the present study, it was demonstrated that high accumulation of 5-ALA-induced PpIX within tumor cells consequently lead to T2 signal intensity enhancement within HGGs; conventional MRI could observe these phenomena. These results suggest that it is possible to use 5-ALA clinically in the metabolic imaging of HGGs using MRI.
A number of previous studies have reported that the normal BBB is impermeable to 5-ALA $(38,39)$, while others have reported that 5-ALA is able to cross the normal BBB to a certain degree at high blood concentrations (40-42). Thus, in the case of HGGs, the BBB breakdown within tumors is considered to serve a permissive role in the uptake and processing of 5-ALA-induced PpIX (43). However, 5-ALA does not perform as well as GBCA in HGG cases. A previous study demonstrated that 5-ALA-induced PpIX fluorescence had a higher diagnostic accuracy compared with GBCA in 
a murine model of infiltrative human glioma (44). Moreover, another prospective study reported that 5-ALA-induced PpIX was superior to GBCA-enhanced intraoperative MRI with regards to its sensitivity and specificity in patients with HGG (8). In addition, 5-ALA-induced PpIX fluorescence was observed in recurrent GBM without contrast enhancement of GBCA following bevacizumab treatment (45). Considering its increased vascular permeability, increased cellular metabolism and a modified tumor microenvironment, 5-ALA-induced PpIX may depict dispersed infiltrative glioma cells more accurately compared with GBCA (46).

The mechanism underlying the enhancement of the T2 signal intensity by 5-ALA-induced PpIX remains unclear. Only one study has attempted to depict experimental gliomas using 5-ALA with MRI in vivo (47). This study demonstrated the complete excretion of 5-ALA-induced PpIX and an increase in intracellular iron $24 \mathrm{~h}$ after 5-ALA administration, and subsequently, a decrease of T2* signal intensity, which is used to depict paramagnetic deoxyhemoglobin, methemoglobin or hemosiderin on MRI (48), in brain tumors (37). Histological evaluation revealed multifocal iron deposits within the tumor adjacent to normal brain tissue in mice $24 \mathrm{~h}$ after 5-ALA treatment (37). 5-ALA does not only accumulate as PpIX in cancer. Recent studies have reported that 5-ALA induced the restoration of oxidative phosphorylation, the suppression of glycolysis, the disruption of the Warburg effect in cancer cells, and the induction of tumor cytotoxic macrophages to the surface of subcutaneous experimental gliomas following 5-ALA administration $(21,49)$. These phenomena may affect iron metabolism in glioma cells $24 \mathrm{~h}$ after 5-ALA administration, and consequently the $\mathrm{T}^{*}$ signal intensity may decrease. In the present study, the T2 signal intensity in HGGs was increased $\sim 3 \mathrm{~h}$ after 5-ALA administration. A high accumulation of 5-ALA-induced PpIX in the tumors was also quantified using HPLC analysis during this period. Therefore, it may be speculated that 5-ALA-induced PpIX is important in the enhancement of T2 signal intensity within tumors. Possible mechanisms underlying PpIX-induced enhancement of T2 signal intensity are as follows. Firstly, relative increase of intracellular content by water insoluble, 5-ALA-induced PpIX may affect enhancement of T2 signal in HGG. Secondly, PpIX is well known for its diamagnetism (50). Thus, accumulated PpIX within the tumor may induce an increase in T2 signal intensity, as well as oxyhemoglobin (51). The present study examined the T2WI of patients following 5-ALA administration only due to the psychophysiological stress that occurs prior to surgery. Therefore, future studies should consider other MRI sequences including T1WI, diffusion imaging, T2*, and intraoperative MRI under general anesthesia.

The current study has several limitations. Firstly, ROIs in T2WIs were obtained from only 4 patients. Thus, future studies should consider the use of a larger sample size. Secondly, the duration of the MRI examination prior to and following administration of 5-ALA was 1 to 8 days. Therefore, the possibility that there was an increase of intratumoral edema that developed during these periods, and affected the enhancement of the T2 signal intensity cannot be excluded. Thus, the duration of the MRI examination should be shortened.

In conclusion, previous studies have focused on the enhancement of the T1 signal intensity using metal-based contrast reagents, including GBCA, in HGG MRI. The present study supports the possibility of using 5-ALA-induced PpIX as a contrast reagent for the enhancement of the T2 signal intensity in conventional MRI for HGGs. At present, the enhancement effect of 5-ALA-induced PpIX is not sufficient for clinical application. However, both the 5-ALA reagent and conventional MRI systems have already been widely used for clinical neurosurgery globally. Therefore, the application of 5-ALA as an MRI contrast reagent is possible, whilst the development of a specific MRI sequence for 5-ALA-induced PpIX is essential.

\section{References}

1. Shapiro WR, Green SB, Burger PC, Mahaley MS Jr, Selker RG, VanGilder JC, Robertson JT, Ransohoff J, Mealey J Jr and Strike TA: Randomized trial of three chemotherapy regimens and two radiotherapy regimens and two radiotherapy regimens in postoperative treatment of malignant glioma. Brain Tumor Cooperative Group Trial 8001. J Neurosurg 71: 1-9, 1989.

2. Stupp R, Mason WP, van den Bent MJ, Weller M, Fisher B, Taphoorn MJ, Belanger $\mathrm{K}$, Brandes AA, Marosi C, Bogdahn U, et al: Radiotherapy plus concomitant and adjuvant temozolomide for glioblastoma. N Engl J Med 352: 987-996, 2005.

3. Westphal M, Ram Z, Riddle V, Hilt D and Bortey E; Executive Committee of the Gliadel Study Group: Gliadel wafer in initial surgery for malignant glioma: Long-term follow-up of a multicenter controlled trial. Acta Neurochir (Wien) 148: 269-275, 2006.

4. Lacroix M, Abi-Said D, Fourney DR, Gokaslan ZL, Shi W, DeMonte F, Lang FF, McCutcheon IE, Hassenbusch SJ, Holland E, et al: A multivariate analysis of 416 patients with glioblastoma multiforme: Prognosis, extent of resection and survival. J Neurosurg 95: 190-198, 2001.

5. Sanai N, Polley MY, McDermott MW, Parsa AT and Berger MS: An extent of resection threshold for newly diagnosed glioblastomas. J Neurosurg 115: 3-8, 2011

6. Laperriere N, Zuraw L and Cairncross G; Cancer Care Ontario Practice Guidelines Initiative Neuro-Oncology Disease Site Group: Radiotherapy for newly diagnosed malignant glioma in adults: A systematic review. Radiother Oncol 64: 259-273, 2002.

7. Wen PY, Macdonald DR, Reardon DA, Cloughesy TF, Sorensen AG, Galanis E, Degroot J, Wick W, Gilbert MR, Lassman AB, et al: Updated response assessment criteria for high-grade gliomas: Response assessment in neuro-oncology working group. J Clin Oncol 28: 1963-1972, 2010.

8. Coburger J, Engelke J, Scheuerle A, Thal DR, Hlavac M, Wirtz CR and König R: Tumor detection with 5-aminolevulinic acid fluorescence and Gd-DTPA-enhanced intraoperative MRI at the border of contrast-enhancing lesions: A prospective study based on histopathological assessment. Neurosurg Focus 36: E3, 2014.

9. Claes A, Idema AJ and Wesseling P: Diffuse glioma growth: A guerilla war. Acta Neuropathol 114: 443-458, 2007.

10. Hutterer M, Hattingen E, Palm C, Proescholdt MA and Hau P: Current standards and new concepts in MRI and PET response assessment of antiangiogenic therapies in high-grade glioma patients. Neuro Oncol 17: 784-800, 2015.

11. Earnest F IV, Kelly PJ, Scheithauer BW, Kall BA, Cascino TL, Ehman RL, Forbes GS and Axley PL: Cerebral astrocytomas: Histopathologic correlation of MR and CT contrast enhancement with stereotactic biopsy. Radiology 166: 823-827, 1988.

12. Wang Y, Alkasab TK, Narin O, Nazarian RM, Kaewlai R, Kay J and Abujudeh HH: Incidence of nephrogenic systemic fibrosis after adoption of restrictive gadolinium-based contrast agent guidelines. Radiology 260: 105-111, 2011.

13. Ishizuka M, Hagiya Y, Mizokami Y, Honda K, Tabata K, Kamachi T, Takahashi K, Abe F, Tanaka T, Nakajima M, et al: Porphyrins in urine after administration of 5-aminolevulinic acid as a potential tumor marker. Photodiagnosis Photodyn Ther 8: 328-331, 2011.

14. Yamamoto J, Yamamoto S, Hirano T, Li S, Koide M, Kohno E, Okada M, Inenaga C, Tokuyama T, Yokota N, et al: Monitoring of singlet oxygen is useful for predicting the photodynamic effects in the treatment for experimental glioma. Clin Cancer Res 12: 7132-7139, 2006.

15. Ishizuka M, Abe F, Sano Y, Takahashi K, Inoue K, Nakajima M, Kohda T, Komatsu N, Ogura S and Tanaka T: Novel development of 5-aminolevurinic acid (ALA) in cancer diagnoses and therapy. Int Immunopharmacol 11: 358-365, 2011. 
16. Yamamoto J, Ogura S, Tanaka T, Kitagawa T, Nakano Y, Saito T, Takahashi M, Akiba D and Nishizawa S: Radiosensitizing effect of 5-aminolevulinic acid-induced protoporphyrin IX in glioma cells in vitro. Oncol Rep 27: 1748-1752, 2012

17. Stummer W, Stocker S, Wagner S, Stepp H, Fritsch C, Goetz C, Goetz AE, Kiefmann R and Reulen HJ: Intraoperative detection of malignant gliomas by 5-aminolevulinic acid-induced porphyrin fluorescence. Neurosurgery 42: 518-526, 1998.

18. Stummer W, Pichlmeier U, Meinel T, Wiestler OD, Zanella F and Reulen HJ; ALA-Glioma Study Group: Fluorescence-guided surgery with 5-aminolevulinic acid for resection of malignant glioma: A randomised controlled multicentre phase III trial. Lancet Oncol 7: 392-401, 2006.

19. Junga CI, Yang JI, Park CH, Lee JB and Park HR: Formation of the Metal Complexes between Protoporphyrin IX and Divalent Metal Cations in the Environment. In: Molecular Environmental Soil Science at the Interfaces in the Earth's Critical Zone. Xu J and Huang PM (eds.) Springer Berlin Heidelberg, Berlin, pp 97-99, 2010.

20. Stummer W, Novotny A, Stepp H, Goetz C, Bise K and Reulen HJ Fluorescence-guided resection of glioblastoma multiforme by using 5-aminolevulinic acid-induced porphyrins: A prospective study in 52 consecutive patients. J Neurosurg 93: 1003-1013, 2000

21. Yamamoto J, Ogura S, Shimajiri S, Nakano Y, Akiba D, Kitagawa T, Ueta K, Tanaka T and Nishizawa S: 5-aminolevulinic acid-induced protoporphyrin IX with multi-dose ionizing irradiation enhances host antitumor response and strongly inhibits tumor growth in experimental glioma in vivo. Mol Med Rep 11: 1813-1819, 2015.

22. Mlkvy P, Messmann H, Pauer M, Stewart JC, Millson CE, MacRobert AJ and Bown SG: Distribution and photodynamic effects of meso-tetrahydroxyphenylchlorin (mTHPC) in the pancreas and adjacent tissues in the Syrian golden hamster. Br J Cancer 73: 1473-1479, 1996.

23. Yamamoto J, Kakeda S, Shimajiri S, Takahashi M, Watanabe K, Kai Y, Moriya J, Korogi Y and Nishizawa S: Tumor consistency of pituitary macroadenomas: Predictive analysis on the basis of imaging features with contrast-enhanced 3D FIESTA at 3T. AJNR Am J Neuroradiol 35: 297-303, 2014.

24. Pierallini A, Caramia F, Falcone C, Tinelli E, Paonessa A Ciddio AB, Fiorelli M, Bianco F, Natalizi S, Ferrante L and Bozzao L: Pituitary macroadenomas: Preoperative evaluation of consistency with diffusion-weighted MR imaging-initial experience. Radiology 239: 223-231, 2006.

25. Watanabe K, Kakeda S, Yamamoto J, Ide S, Ohnari N Nishizawa $\mathrm{S}$ and Korogi Y: Prediction of hard meningiomas: Quantitative evaluation based on the magnetic resonance signal intensity. Acta Radiol 57: 333-340, 2016.

26. Louis DN, Ohgaki H, Wiestler OD, Cavenee WK, Burger PC, Jouvet A, Scheithauer BW and Kleihues P: The 2007 WHO classification of tumours of the central nervous system. Acta Neuropathol 114: 97-109, 2007.

27. Kreisl TN, Kim L, Moore K, Duic P, Royce C, Stroud I, Garren N, Mackey M, Butman JA, Camphausen K, et al: Phase II trial of single-agent bevacizumab followed by bevacizumab plus irinotecan at tumor progression in recurrent glioblastoma. J Clin Oncol 27: 740-745, 2009

28. Hasselbalch B, Lassen U, Hansen S, Holmberg M, Sørensen M, Kosteljanetz M, Broholm H, Stockhausen MT and Poulsen HS: Cetuximab, bevacizumab, and irinotecan for patients with primary glioblastoma and progression after radiation therapy and temozolomide: A phase II trial. Neuro Oncol 12: 508-516, 2010.

29. Prados M, Cloughesy T, Samant M, Fang L, Wen PY, Mikkelsen T, Schiff D, Abrey LE, Yung WK, Paleologos N, et al: Response as a predictor of survival in patients with recurrent glioblastoma treated with bevacizumab. Neuro Oncol 13: 143-151, 2011.

30. Huang RY, Rahman R, Hamdan A, Kane C, Chen C, Norden AD, Reardon DA, Mukundun S and Wen PY: Recurrent glioblastoma: Volumetric assessment and stratification of patient survival with early posttreatment magnetic resonance imaging in patients treated with bevacizumab. Cancer 119: 3479-3488, 2013.

31. Henson JW, Ulmer S and Harris GJ: Brain tumor imaging in clinical trials. AJNR Am J Neuroradiol 29: 419-424, 2008.

32. Schmainda KM, Prah M, Connelly J, Rand SD, Hoffman RG, Mueller W and Malkin MG: Dynamic-susceptibility contrast agent MRI measures of relative cerebral blood volume predict response to bevacizumab in recurrent high-grade glioma. Neuro Oncol 16: 880-888, 2014
33. Ellingson BM, Kim HJ, Woodworth DC, Pope WB, Cloughesy JN, Harris RJ, Lai A, Nghiemphu PL and Cloughesy TF: Recurrent glioblastoma treated with bevacizumab: Contrast-enhanced T1-weighted subtraction maps improve tumor delineation and aid prediction of survival in a multicenter clinical trial. Radiology 271: 200-210, 2014.

34. Dunet V, Rossier C, Buck A, Stupp R and Prior JO: Performance of $18 \mathrm{~F}$-fluoro-ethyl-tyrosine (18F-FET) PET for the differential diagnosis of primary brain tumor: A systematic review and Metaanalysis. J Nucl Med 53: 207-214, 2012.

35. Nihashi T, Dahabreh IJ and Terasawa T: PET in the clinical management of glioma: Evidence map. AJR Am J Roentgenol 200: W654-W660, 2013.

36. Hutterer M, Nowosielski M, Putzer D, Jansen NL, Seiz M, Schocke M, McCoy M, Göbel G, la Fougère C, Virgolini IJ, et al: [18F]-fluoro-ethyl-L-tyrosine PET: A valuable diagnostic tool in neuro-oncology, but not all that glitters is glioma. Neuro Oncol 15: 341-351, 2013.

37. Galldiks N, Rapp M, Stoffels G, Fink GR, Shah NJ, Coenen HH, Sabel M and Langen KJ: Response assessment of bevacizumab in patients with recurrent malignant glioma using [18F]Fluoroethyl-L-tyrosine PET in comparison to MRI. Eur J Nucl Med Mol Imaging 40: 22-33, 2013

38. Garcia SC, Moretti MB, Garay MV and Batlle A: Delta-aminolevulinic acid transport through blood-brain barrier. Gen Pharmacol 31: 579-582, 1998.

39. Terr L and Weiner LP: An autoradiographic study of delta-aminolevulinic acid uptake by mouse brain. Exp Neurol 79: 564-568, 1983

40. McGillion FB, Thompson GG, Moore MR and Goldberg A: The passage of delta-aminolaevulinic acid across the blood-brain barrier of the rat: Effect of ethanol. Biochem Pharmacol 23: 472-474, 1974.

41. Ennis SR, Novotny A, Xiang J, Shakui P, Masada T, Stummer W, Smith DE and Keep RF: Transport of 5-aminolevulinic acid between blood and brain. Brain Res 959: 226-234, 2003.

42. Gibson SL, Havens JJ, Foster TH and Hilf R: Time-dependent intracellular accumulation of delta-aminolevulinic acid, induction of porphyrin synthesis and subsequent phototoxicity. Photochem Photobiol 65: 416-421, 1997.

43. Stummer W, Reulen HJ, Novotny A, Stepp H and Tonn JC: Fluorescence-guided resections of malignant gliomas-an overview. Acta Neurochir Suppl 88: 9-12, 2003.

44. Samkoe KS, Gibbs-Strauss SL, Yang HH, Khan Hekmatyar S, Jack Hoopes P, O'Hara JA, Kauppinen RA and Pogue BW: Protoporphyrin IX fluorescence contrast in invasive glioblastomas is linearly correlated with Gd enhanced magnetic resonance image contrast but has higher diagnostic accuracy. J Biomed Opt 16: 096008, 2011.

45. Wachter D, Kallenberg K, Wrede A, Schulz-Schaeffer W, Behm T and Rohde V: Fluorescence-guided operation in recurrent glioblastoma multiforme treated with bevacizumab-fluorescence of the noncontrast enhancing tumor tissue? J Neurol Surg A Cent Eur Neurosurg 73: 401-406, 2012.

46. Collaud S, Juzeniene A, Moan J and Lange N: On the selectivity of 5-aminolevulinic acid-induced protoporphyrin IX formation. Curr Med Chem Anticancer Agents 4: 301-316, 2004.

47. Cho HR, Kim DH, Kim D, Doble P, Bishop D, Hare D, Park CK, Moon WK, Han MH and Choi SH: Malignant glioma: MR imaging by using 5 -aminolevulinic acid in an animal model. Radiology 272: 720-730, 2014

48. Chavhan GB, Babyn PS, Thomas B, Shroff MM and Haacke EM: Principles, techniques, and applications of T2*-based MR imaging and its special applications. Radiographics 29: 1433-1449, 2009

49. Sugiyama $Y$, Hagiya $Y$, Nakajima $M$, Ishizuka $M$, Tanaka $T$ and Ogura S: The heme precursor 5-aminolevulinic acid disrupts the Warburg effect in tumor cells and induces caspase-dependent apoptosis. Oncol Rep 31: 1282-1286, 2014.

50. Desideri A, Caccuri AM, Polizio F, Bastoni R and Federici G: Electron paramagnetic resonance identification of a highly reactive thiol group in the proximity of the catalytic site of human placenta glutathione transferase. J Biol Chem 266: 2063-2066, 1991.

51. Hiwatashi A, Kinoshita T, Moritani T, Wang HZ, Shrier DA, Numaguchi Y, Ekholm SE and Westesson PL: Hypointensity on diffusion-weighted MRI of the brain related to T2 shortening and susceptibility effects. AJR Am J Roentgenol 181: 1705-1709, 2003. 